\title{
Towards a Better Understanding of Dark Matter Halo Structures around Galaxies
}

\author{
Melissa Wheeler \\ Physics Department \\ University of Wisconsin- La Crosse, La Crosse, WI
}

\begin{abstract}
There is substantial evidence that the stars, dust, and gas visible in galaxies are enveloped in "halos" of unseen dark matter. The goal of this research is to explore the effects varying initial conditions have on final equilibrium distributions of simulated dark matter halo density and velocity, and to assess how well analytical models fit these data. In particular, we pay special attention to the velocity distributions of our models, something often overlooked in other studies. Eighty separate computer simulations representing dark matter halos were made under various initial conditions, involving two halo types and three variables. Analytical density and velocity models were fitted to the 80 halos, including de Vaucouleurs, Plummer, generalized NavarroFrenk-White, and non-extensive thermodynamics models. Statistical chi-squared $\left(\chi^{2}\right)$ tests, quantifying how well a model fits data, were applied. Relative $\chi^{2}$ values for each model were plotted, reflecting which analytical models performed best for various simulations.
\end{abstract}

\section{Background}

While it may sometimes seem as if science has the universe pretty much figured out, that is far from the truth. One of the most mysterious aspects of the universe discovered over the last century is the apparent necessity of dark matter. By the term "dark matter", we mean an unidentified type of matter that appears to give off little or no light (hence the "dark"), yet whose gravity influences the "light" matter that we can see, like stars and galaxies. The need for dark matter was first highlighted in the 1930s by astronomer Fritz Zwicky. According to the laws of physics, the observed motion of galaxies in clusters is incompatible with the mass inferred from the light present (Zwicky 1933, 1937). Galaxy clusters contain hundreds to thousands of individual galaxies that move under the influence of their combined gravity, and more details on how they can be used to investigate dark matter are given below. In the intervening decades, a large body of evidence for the existence of this unseen matter has been gathered. Current measurements and calculations suggest that the mass of dark matter in galaxies is at least 10 times greater than the mass in the form of stars and gas.

When we look at our own Milky Way galaxy (called the Galaxy for short), it is logical to infer that the majority of the Galaxy's mass must lie in the center, where the high brightness shows that the mass of the stars is concentrated. Given this, we would expect the speed of stars orbiting the Galactic center to decrease with distance as the force of gravity from the center decreases. This relationship between orbital speed, distance, and gravitational force follows from the understanding that gravity acts as a centripetal force in the Galaxy.

$$
G \frac{M m}{r^{2}}=m \frac{v^{2}}{r}
$$

where $G$ is Newton's gravitational constant, $M$ is the mass of the Galaxy interior to distance $r, m$ is the mass of a star, and $v$ is the orbital speed of the star. Gravity continually changes the direction of stars' velocities so that they follow a circular path in the Milky Way.

We gratefully acknowledge the National Space Grant College and Fellowship Program and the Wisconsin Space Grant Consortium for supporting this research. 
This relationship holds for the planets in our solar system. Since the sun contains far more mass than all the planets combined, $M$ in this case is just the mass of the sun. Planets that are further from the sun take longer to complete one orbit of it (e.g. Earth takes one year to complete an orbit, whereas Jupiter takes almost 12 years). However, this is not what we see with stars orbiting in galaxies. Plots of orbital speed versus orbital distance are called rotation curves. The rotation curve of our solar system drops off as distance from the sun increases (Figure 1), illustrating the prediction from the above equation.

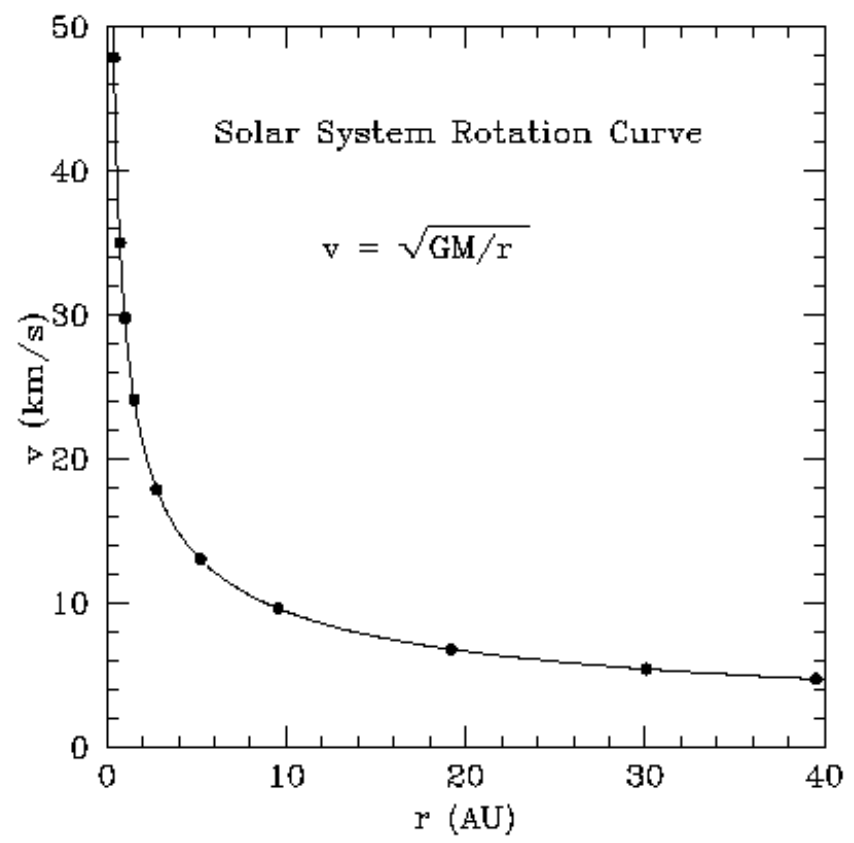

Figure 1. Velocity vs. distance graph showing predicted orbital speeds for planets in the solar system. As distance from the sun increases, velocity of the orbiting body decreases. Taken from bustard.phys.nd.edu/Phys171/lectures/dm.html .

When the orbital speed of stars (or gas) is graphed against their average distance from the center of the Galaxy, the resulting rotation curve is relatively flat (Figure 2), showing that the orbital speed remains large far from the galactic center. This implies that most of the Galaxy's mass lies not at the center, but surrounds the visible Galaxy in what is referred to as a halo, which is usually assumed to be spherical. Since very little radiation can be detected from this halo, it is assumed to be composed of dark matter. Detailed analysis of this rotation curves suggests that the Galactic dark matter mass is roughly 10 times the amount of mass in all the stars in the Milky Way.

Evidence for dark matter is also seen elsewhere in the universe. In other spiral galaxies, we can also use rotation curves to estimate their total masses. The figure below shows the orbital speeds of stars and gas in another galaxy as a function of distance from that galaxy's center. As with the Milky Way, the rotational speed does not decrease as expected far from the central mass concentration. 


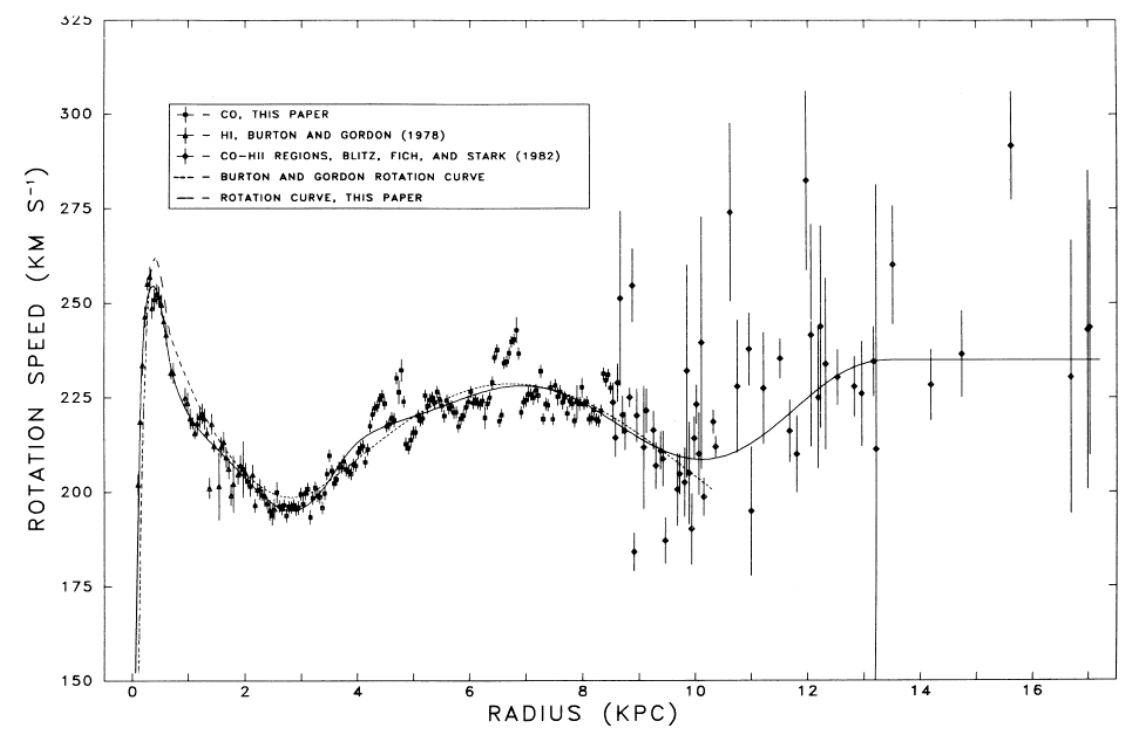

Figure 2. The rotation curve for the Milky Way Galaxy, showing a relatively flat result, which signifies fairly constant, large orbital speeds as distance from the Galactic center increases. Taken from web.njit.edu/ gary/202/Lecture25.html. Data taken from Clemens (1985).

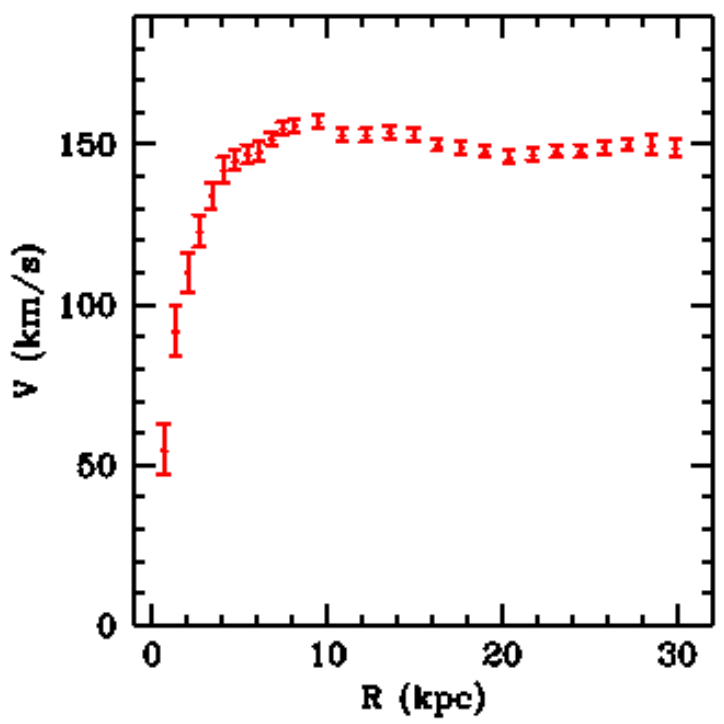

Figure 3. Graph of orbital velocity against distance from galactic center of a spiral galaxy, showing a relatively flat rotation curve. Image taken from astro.berkeley.edu/ mwhite/darkmatter/rotcurve.html; graph data from Begeman (1989).

The galaxy's total mass is found by using the law of gravity and the orbital velocities of stars and gas clouds. The luminosity, i.e. how much light the stars produce, can be used to infer the amount of mass that is in the form of stars. For example, we know how much light is emitted by our sun and we know its mass. Decades of astronomy have fine-tuned relationships between the light and mass of a wide variety of stars that exist in galaxies. If the total mass is greater than the mass attributed to the stars (and gas), the rest is inferred to be dark matter. Just like with the Milky Way, further analysis also suggests that other spiral galaxies contain at least 10 times as 
much dark matter as they do stars; this means that the composition of a typical spiral galaxy is about $10 \%$ normal matter like stars and $90 \%$ dark matter.

In elliptical galaxies, we cannot use rotation curves because the stars' motions are more disorganized, so we measure the combined Doppler shifts of the stars (which tell us if they are moving towards or away from us and how fast) in the light the galaxy emits. These shifts change the light from a narrow spectral line of a certain wavelength to a broadened line that covers a range of wavelengths; the wider the spectral line, the faster the stars are moving. When spectral lines from different areas of the galaxy are compared, it is seen that the speeds of the stars remain fairly constant as we get farther from the galaxy's center, just as with spiral galaxies. While the analysis of the motions of stars in elliptical galaxies is not as simple as in spiral galaxies, it is commonly accepted that elliptical galaxies also contain large amounts of dark matter.

Concerning clusters of galaxies, there are three different ways of measuring the mass of the cluster, each of which gives more evidence for dark matter. First, the speeds of galaxies that orbit the cluster center are measured; this is the method that led to Zwicky's initial proposition of the existence of dark matter. After finding the average orbital velocity of the galaxies within the cluster and using the laws of physics to estimate the mass if the cluster, the cluster's mass is compared to its luminosity. Again, the stellar and gas mass necessary to generate that luminosity is found to be far less than the mass measured from galaxy speeds. The second method utilizes observations of X-rays from hot gas that resides between galaxies in a cluster. This gas can contain a great deal of mass, at times outweighing even the total mass in stars in the galaxies in the cluster. The temperature of this gas depends on the overall mass of the cluster, as the gas pressure outward is balance by the inward pull of the cluster's gravity. As temperature reflects the kinetic energy of the particles and therefore the average speed of the particles, we can use these speeds to find the total mass of the cluster. These results agree with those of the first method, in that even with the added mass of the hot gas, the amount of dark matter is up to 50 times as great as the mass of the stars in the cluster's galaxies (Binney \& Tremaine 1987). A third way to measure the mass of a galaxy cluster is by using gravitational lensing, which is especially useful because it does not use Newton's laws of gravity and so allows another method of checking results. Predicted by Einstein's general theory of relativity, gravitational lensing occurs because masses distort space-time; massive objects can bend light beams as they pass, instead of allowing the photons to travel in a straight line from their source. The angle of the bending depends on the mass of the object acting as a gravitational lens, so we can measure the mass by looking at how strongly the light paths are distorted. These results generally agree with those obtained by the first two methods, all suggesting that galaxy clusters contain a large amount of dark matter.

Researchers are yet unsure exactly what dark matter may be made of. It is believed that it falls into two categories: baryonic dark matter (i.e. "normal" matter made of protons, neutrons, etc. that simply does not emit very much light) and non-baryonic matter made of more extraordinary particles. Baryonic dark matter includes brown dwarfs, which are celestial objects too small to generate nuclear fusion to shine like a normal star, and a faint red star type; it is possible that many such small, dim objects (sometimes called massive compact halo objects or MACHOs) continue to roam in galaxy halos, but studies have shown that they do not exist in large enough 
numbers to compose all of the dark matter. Non-baryonic dark matter is thought to consist of subatomic particles similar to neutrinos but heavier; these hypothetical particles are called weakly interacting massive particles, or WIMPs. WIMPs would have no electrical charge (and therefore not be able to emit any electromagnetic radiation), and could only interact with other matter through gravity and the weak force, making them difficult to detect directly. As a galaxy formed and normal matter collapsed into a flattened disk due to gravity and the loss of orbital energy through collisions, these particles would rarely interact with other particles, and so maintain their farther out orbits, forming the halo. Many researchers think that WIMPs may compose the majority of dark matter.

If dark matter does not exist, then there must be something wrong with our current understanding of the laws of physics and gravity. Many scientists have attempted to come up with alternative theories of gravity that would rule out the need for the existence of unseen dark matter, though none have successfully been able to do so while still explaining many other observations that the dark matter-based theories can explain. There is much evidence for the existence of dark matter, and some observations that are difficult to explain without it. For example, the "bullet" cluster (Clowe et al. 2006) is often referred to as the most compelling evidence for dark matter. In this case, two galaxy clusters collided at some point; the stars and galaxies of the clusters simply passed each other by, but the gas violently collided. Gravitational lensing measurements suggest that the majority of the mass in the clusters is centered around the galaxies, as predicted by a dark matter picture.

Much remains unknown concerning the nature and behavior of dark matter. Our work involved computer simulations that aimed to investigate the behavior of self-gravitating systems, a category into which dark matter halo structures fall, under a variety of initial conditions. The simulations were given initial positions and velocities for particles representing masses of dark matter. The computer then evolved the subsequent motions of the particles as acted on by gravity, resulting in data on the final equilibrium distribution of the system. We created a total of 80 simulations of 100,000 particles that span a range of initial conditions, which will be described in greater detail in the next section. Analysis has been done on the density and velocity profiles for the halos, and several analytical models that have been used to describe dark matter distributions have been fitted to the data. We were specifically interested in the performance of non-extensive thermodynamic models, a class of analytical models that has been discussed for dark matter halos (e.g., Kronberger et al. 2006, Barnes et al. 2007). Analysis, such as the statistical chi-squared test, has been done to determine how well each model fits the data for specific initial conditions. Also, several types of plots have been created to illustrate different aspects of the comparison of models to the data and to each other. These will be explained and findings described in Results section.

\section{Methods}

This research used a computer to create simulations of particles representing masses of dark matter. These simulations that involve large numbers of massive particles being influenced by gravity are known as $N$-body models and are approximately collisionless. The particles' individual motions are affected by the gravity of the system as a whole, not by interactions with their close neighbors. We utilized $N$-body systems of 100,000 particles to simulate the behavior of dark matter particles as they interact gravitationally within the halo. We were interested in the 
final equilibrium state of the halo and how the density and velocity distributions of particles have settled, i.e. where they end up and how they are moving. Each simulation was evolved from a certain set of initial conditions involving three variables and two particle distribution types.

We have used a variety of initial conditions in the creation of our simulations to compare the effect such differences may have on the analytical model fits. First, two types of model halos were simulated --- "single" and "clumpy". In single simulations, the particles were evenly and smoothly distributed throughout the system, and individual particles were given random initial velocities. Particles in clumpy halos were bound by gravity in groups, or clumps. This affects how the particles interacted within their own clump and with other clumps in the system, and influenced the final shape of the halo. We will discuss in more detail the clumpy models first.

Within our initial conditions for the clumpy halo models there were three variables, resulting in a total of 60 clumpy simulations. The first variable deals with the initial density $\rho$ profile, which determines whether the particles are likely to be found near the center of the system or more uniformly spread throughout. The initial density profile followed $\rho \propto 1 / r$ (cuspy) or $\rho \propto \mathrm{e}^{-r^{2}}$ (Gaussian). The second variable is the virial ratio, represented by $Q$ and ranging from $0.10,0.20$, ..., 1.00. The virial ratio is defined as twice the kinetic energy divided by the potential energy. A low value of $Q$ means the system has little kinetic energy, which leads to low particle speeds and initial collapse of the system. Systems with high values of $Q$ do not collapse as notably as those with low $Q$ values, as the particles have more kinetic energy and higher speeds initially. The clumpy models also had three different temperature fractions, or $T$ values: $0.0,0.5$, or 1.0 . A system with $T=1.0$ is said to have "cold" conditions, where all particles in the clump move together with the clump center-of-mass. $T=0.0$ is a "hot" system, and clump particles have random initial velocities and center-of-mass velocities are zero. A system with $T=0.5$ is a "warm", intermediate system. Each clumpy simulation was given a specific density profile, $Q$ value, and $T$ value, requiring 60 separate models to span the clumpy initial condition parameter space.

Single halo models, where particles were distributed evenly throughout the system, resembled clumpy $T=0.0$ systems in that all particles had random initial velocities. Since they were not bound gravitationally in clumps, single simulations did not include a temperature fraction $T$ variable. The cuspy and Gaussian density profile types and range of $Q$ values remained the same, leading to 20 different single halo simulations. This gave us a grand total of 80 dark matter halo evolutions spanning a variety of initial conditions.

Once the simulated dark matter halos had been evolved, output data were given on the final state of the halo after it had reached dynamic equilibrium. We were concerned with the end-state of the density and velocity profiles, i.e. where the particles end up and how they are moving. Plots of these data were created, and then analytical models were fitted to the data. Computer code found the best-fit parameters for each of the de Vaucouleurs, Plummer, Navarro-Frenk-White (NFW), and non-extensive models to each of the 80 halo simulations. From these density profile fits, velocity profiles were estimated and also compared to the data. Statistical chi-squared $\left(\chi^{2}\right)$ tests, quantifying how well a model fits data, were applied to the analytical fits. From these values plots of the relative $\chi^{2}$ values for each model were plotted, reflecting which analytical models performed best for various simulations. Our particular focus was on the performance of 
the non-extensive thermodynamics model in fitting the data and how it compares to the other models. We also paid special attention to the velocity distributions of our models, something often overlooked in other studies.

\section{Results}

From these relative $\chi^{2}$ values, it can be seen which analytical models fit the simulations best for various initial conditions. Plots were created for each set of clumpy and single halos, differing in initial density $\rho$ profile and/or temperature fraction $T$. These graphs show how the analytical models performed for each set as $Q$ varies; each includes data for 10 simulations. Density, velocity and combined $\chi^{2}$ values were studied. Every analytical model's $\chi^{2}$ is divided by the lowest $\chi^{2}$ value for each $Q$. Therefore the best-fitting model at every $Q$ is given a relative $\chi^{2}$ of 1 ; the true $\chi^{2}$ value for the model is likely higher. Several plots of relative $\chi^{2}$ values are included as examples. These plots reflect the results for the group of 10 simulations of clumpy halos with cuspy initial density and $T=1.0$, showing relative model performance as $Q$ changes.

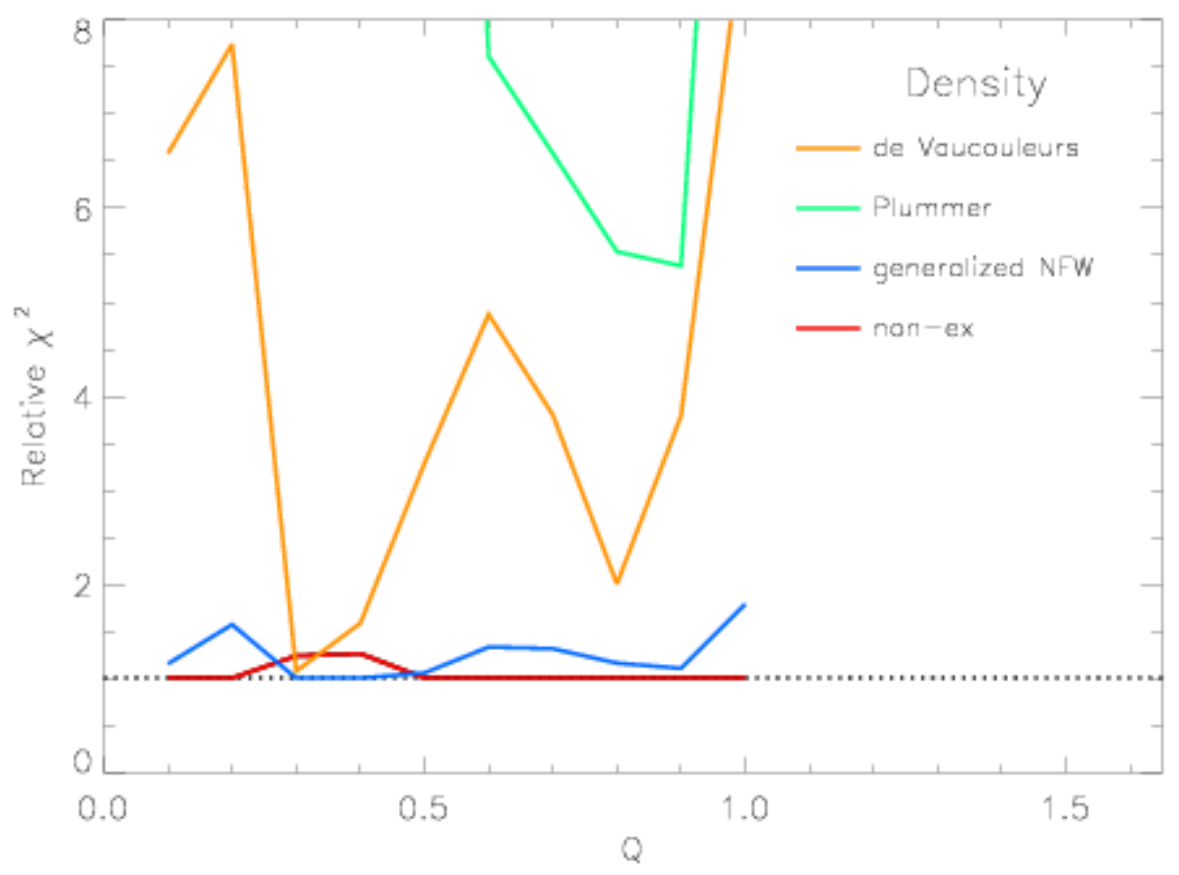

Figure 4.- The relative $\chi^{2}$ density results for the set of clumpy $T=1.0$ simulations with cuspy initial density. The density data obtained from the clumpy, cuspy $T=1.0$ simulations are best fit by the non-extensive analytical model for most values of $Q$, particularly the higher ones. 


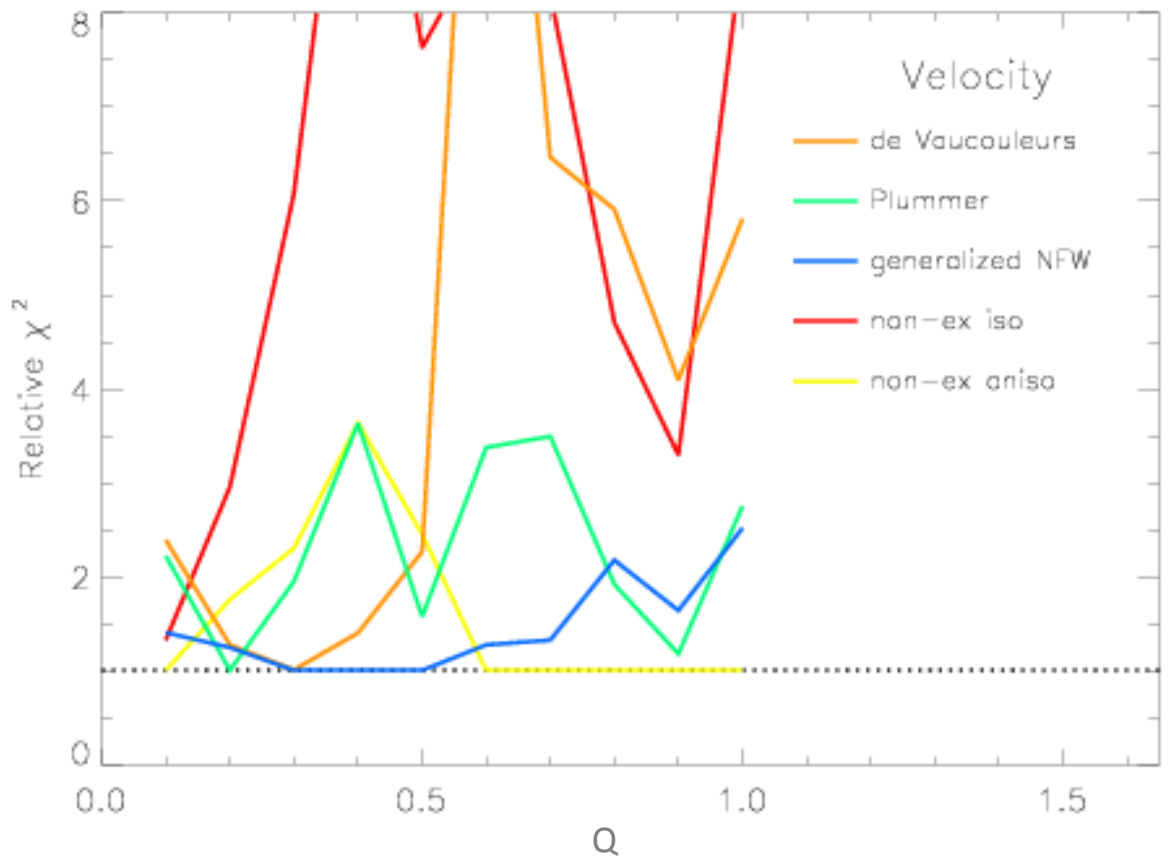

Figure 5.- The relative $\chi^{2}$ velocity results for the set of clumpy, cuspy $T=1.0$ simulations. Two possible nonextensive velocity profiles - isotropic (red) and anisotropic (yellow) - are given. The velocity $\chi^{2}$ results for this set of simulations show that the non-extensive anisotropic velocity profile described the data best at high values of $Q$, while the NFW model did better for mid- to low-range $Q$ values.

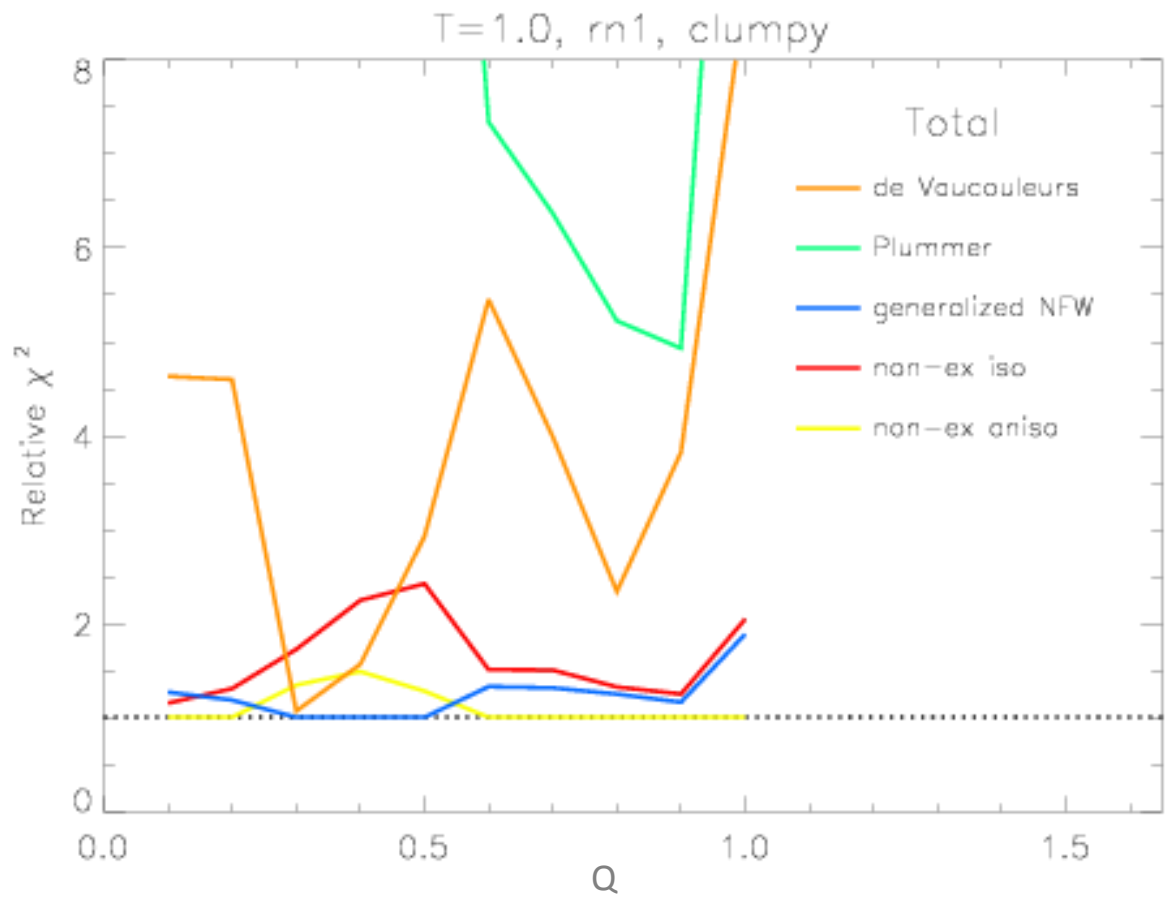

Figure 6.- The total relative $\chi^{2}$ comparison, combining both the density and velocity $\chi^{2}$ results for the clumpy, cuspy (rn1) $T=1.0$ simulations. The non-extensive model values are found combining the single density fit $\chi^{2}$ values with each of the isotropic and anisotropic velocity fits. 
As demonstrated by these plots, for this particular set of simulations (clumpy, cuspy $T=1.0$ ), the non-extensive thermodynamics model (allowing for anisotropic velocities) was found to have the relative best fits for very low and mid- to high-range values of $Q$, while the gNFW model performed best for $Q=0.30-0.50$.

For the other clumpy halos with cuspy initial densities, the family of clumpy, cuspy $T=0.5$ simulations were found to be fit best overall by the non-extensive model for $Q \geq 0.40$, though the Plummer model was a slightly better density fit at $Q=0.70$. Both the non-extensive isotropic and anisotropic velocity profiles were better fits at high $Q$ values, with isotropic doing slightly better at the higher values. The $T=0.0$ clumpy, cuspy halos also showed good fits by the non-extensive model above $Q=0.30$, with the exception of $Q=0.5$ where Plummer had a better density fit.

Clumpy halos with Gaussian initial density and $T=1.0$ were actually fit better by the gNFW model for 6 of the simulations, in some cases due to a better velocity fit than the non-extensive. The non-extensive model, particularly when allowing for anisotropic velocities, performed best toward the high $Q$ values. The set of clumpy, Gaussian $T=0.5$ simulations had density profiles fit best almost completely by the non-extensive model, and best non-extensive velocity fits at higher $Q$ values. The combined results show this same model fitting best for mid- to high-range $Q$ values, favoring the anisotropic velocity profile. Finally, the clumpy, Gaussian $T=0.0$ family of halos was best fit by the non-extensive (anisotropic) model for $Q \geq 0.30$ except for the $Q=0.60$ and $Q=0.70$ simulations, where the Plummer model had a better relative total $\chi^{2}$.

Similar results were seen with the single halo simulations. The single halos only involved two variables, initial density $\rho$ profile and virial ratio $Q$. The set of single, cuspy halos were described best overall by the non-extensive model (allowing for anisotropic velocities) with only one exception $(Q=0.50$, Plummer model). Density was best fit at every $Q$ by the non-extensive model, and the anisotropic non-extensive velocity model performed best at higher $Q$ values. Single halos with Gaussian initial density showed best total fits from the anisotropic nonextensive model for middle and high $Q$ values, with the Plummer model fitting better at $Q=0.60$ and $Q=0.70$. The de Vaucouleurs model provided the best velocity fit for the lower half of the $Q$ range.

\section{Conclusions}

Overall, while the best-fit model varied for differing initial conditions, there are trends in the data. We find that the non-extensive models generally provided the relative best fits to our data for density, especially as $Q$ increases. The de Vaucouleurs model performed better with low values of $Q$ for velocity distributions; however, it did not fit simulation density distributions well. The Plummer model was generally a very poor fit for low $Q$ halo densities, but tended to improve in the mid-high $Q$ range. In many cases this model performed second-best to the nonextensive. The generalized NFW model fit the data better at some lower $Q$ values, and its overall best performance- though not necessarily with the lowest $\chi^{2}$ values- was with the clumpy $T=1.0$ halos. The anisotropic non-extensive model tended to fit the velocity profiles better than the isotropic and other models for high $Q$ values. Given this, our research shows that the nonextensive model in general provides the relative best fit to these data, particularly for higher values of $Q$. 
In both single and clumpy simulations, there was more variation in model performance at lower $Q$. This is likely due to the fact that systems with a low virial ratio have little kinetic energy, and so initially collapse violently. The resulting halo distributions are less "well-behaved" than their higher- $Q$ counterparts, making it more difficult for analytical models to find a good fit. The nonextensive model did not always provide a relative best fit for lower $Q$ values, especially for the velocity distributions, and is overall more suitable for higher $Q$ values. It is also worth noting that a model with the lowest $\chi^{2}$ value is not necessarily an excellent fit to the data; it simply resulted in a better fit than the other models.

This research has helped lay a ground work for further investigation into the appropriateness of these analytical models to describe dark matter halo distributions, particularly the non-extensive thermodynamics model. We may in the future investigate cosmological models that include Hubble expansion. As the simulations we have run are collisionless, another possible path is to allow collisions and see if the non-extensive model continues to do well in describing that data.

\section{References}

Barnes, E., Williams, L., Babul, A., Dalcanton, J. 2007, ApJ, 655, 847

Begeman, K. 1989, A\&A, 223, 47

Binney, J., Tremaine, S. 1987, Galactic Dynamics, Princeton Univ. Press, Princeton, NJ

Clemens, D. 1985, ApJ, 295, 422

Clowe, D., Bradač, M., Gonzalez, A., Markevitch, M., Randall, S., Jones, C., Zaritsky, D. 2006, ApJ, 648, 109L

Kronberger, T., Leubner, M., van Kampen, E. 2006, A\&A, 453, 21

Zwicky, F. 1933, H. Phys. A., 6, 110

Zwicky, F. 1937, ApJ, 86, 217 